\title{
CLUSTERS IN PREHEATING SIMULATION AND ITS EFFECT ON CMB SPECTRUM
}

\author{
KAI-YANG LIN ${ }^{1,2}$, TAK-PONG WOO ${ }^{1}$, YAO-HUAN TSENG ${ }^{1}$, LIHWAI LIN $^{1} \&$ TZIHONG $^{2}$ \\ CHIUEH ${ }^{1,2}$ \\ 1. Department of Physics, National Taiwan University, Taipei, Taiwan \\ 2. Institute of Astronomy and Astrophysics, Academia Sinica, Taipei, Taiwan \\ kylin@asiaa.sinica.edu.tw
}

\begin{abstract}
We report an instantaneous heating scheme at $z=2$ that can produce bound objects obeying the observed mass-temperature and luminosity-temperature relations. Such heating reduces the Sunyaev-Zeldovich (SZ) flux by a factor $3-2$ on scale of $4-6$ arcminutes. It exacerbates the need for the matter-fluctuation normalization $\sigma_{8}$ to assume an exceedingly high value $(\sim 1.2)$, if the several-arcminute excess anisotropy detected by the CBI experiment is entirely caused by the SZ effect of inter-galactic hot gas. By contrast, we find that the several-arcminute excess detected by ACBAR can be consistent with the preheated SZ signals of $\sigma_{8}=1$.
\end{abstract}

Keywords: cosmology: theory; cosmic microwave background; intergalactic medium.

\section{Introduction}

In the hierarchical structure formation model of cold dark matter, gravitationally bound objects obey similarity scaling relations ${ }^{1}{ }^{2}$. However, the observed X-ray luminosity-temperature $(L-T)$ relation shows that the cluster temperature appears considerably higher than the scaling $T \propto L^{1 / 2}$, indicates, more so for low-mass clusters than for high-mass clusters ${ }^{345}$. Although the observed mass-temperature $(M-T)$ relation roughly obeys $T \propto M^{2 / 3}$, the temperature is still found higher by $30 \%$ or so than expected from adiabatic structure formation processes ${ }^{6}$. These evidences suggest certain non-gravitational energy injection to be operative. One candidate of heat sources is the outflows from Active Galactic Nucleus (AGN) ${ }^{7}$ 8. Investigators have tested various schemes for heat injection at $z \geq 4^{9} 101112$. Although the observed $L-T$ relation may be recovered, they failed in producing the observed $M-T$ relation.

Parallel to the developments in cluster physics, the CBI ${ }^{13}$ experiment the ACBAR ${ }^{14}$ experiment independently discovered a higher level of temperature anisotropy than anticipated by, around the harmonic mode number $2000<l<3000$, in cosmic-microwave-background (CMB) radiation. This feature was attributed to the Sunyaev-Zeldovich (SZ) effects ${ }^{15}{ }^{16}$. To account for the high level of SZ signals, the value of $\sigma_{8}$ was estimated, by a joint analysis, to be unity, though a slightly larger $\sigma_{8}$ fits the CBI data better. On the other hand, the cluster abundance study of 
the Sloan Digital Sky Survey (SDSS) finds that $\sigma_{8} \Omega_{m}^{0.6}=0.35 \pm 0.03{ }^{17}$, consistent with results of X-ray cluster abundance (See for example Ref. 18 and references therein). The extreme value $\sigma_{8}=1$ thus yields a low matter-density parameter, $\Omega_{m}=0.175 \pm 0.025$. If the SZ effect indeed accounts for the high- $l$ excess of CBI and ACBAR, preheating will exacerbate the awkward situation of the already large $\sigma_{8}$. Past works have indicated that preheating reduces the SZ flux ${ }^{10} 919$. These works were, however, unable to recover both observed $M-T$ and $L-T$, rendering the reduction of SZ flux not amenable to X-ray calibration. Motivated by the evidences of late preheating around $z=2^{20}{ }^{21}$, we study a local heating scheme, which recovers both observed $M-T$ and $L-T$ relations.

\section{2. $M-T$ and $L-T$ Relations in Simulation}

We ran cosmological hydrodynamic simulations with the GADGET code ${ }^{22}$ for both $\sigma_{8}=0.94$ and 1 cases in a periodic box of comoving size $100 h^{-1} \mathrm{Mpc}$. The simulations contain $256^{3}$ dark matter particles and $256^{3}$ gas particles, with the gravitational softening length being $20 h^{-1} \mathrm{kpc}$ and the mass resolution $4.8 \times 10^{9} h^{-1} M_{\odot}$ for the dark matter and $0.83 \times 10^{9} h^{-1} M_{\odot}$ for the gas. All runs start at $z=100$ with the same initial conditions for $\left(n_{s}, \sigma_{8}, \Omega_{m}, \Omega_{b}, \Omega_{\Lambda}, h\right)=(1,0.94,0.34,0.05,0.66,0.66)$.

Heat injection occurs at $z=2$ and assumes a density-dependent form,

$$
\Delta u=u_{0} \exp \left[-\frac{\rho_{b 0}}{\rho_{b}}\right],
$$

where $\rho_{b}$ and $\rho_{b 0}$ are the local and background baryon densities respectively, and $u$ is the specific internal energy. This form of heating ensures the highest-density region to receive a constant specific energy $u_{0}$ while the lowest density region no heat. We find the best value of $u_{0}=7 \mathrm{keV} /$ baryon, or equivalently $0.75 \mathrm{keV} /$ baryon on average. Since our heating occurs late in time and since a present-day cluster of $10 \mathrm{keV}$ is still deficient in x-ray luminosity (c.f. Fig.1(b) below), we find this amount of local heat injection reasonable.

Throughout this work, emission-weighted temperature is used. Figure 1(a) compares both adiabatic and preheating $M_{500}-T_{500}$ relation from our $\sigma_{8}=0.94$ simulation with the fitting formula for adiabatic simulations given by Evrard, Metzler \& Navarro (1996) ${ }^{23}$ and with the fitting formula of HIFLUGCS data given by Finoguenov, Reiprich, \& Böhringer (2001) ${ }^{6}$. The $M_{500}-T_{500}$ relation of the adiabatic simulation agrees fairly with that of Evrard et al. ${ }^{23}$, but both yield too low a temperature in bound objects. On the other hand, our preheating simulation yields an $M_{500}-T_{500}$ relation agreeing very well with the observations.

A constant metalicity, 0.3 times the solar value, is adopted here to compute the X-ray bolometric luminosity. Figure $1(\mathrm{~b})$ shows $L_{500}$ against $T_{500}$. Also shown are the observed $L_{500}$ and $T_{500}$ compiled by Arnaud \& Evrard $(1999)^{24}$, Helsdon $\&$ Ponman $(2000)^{25}$ and Markevitch $(1998)^{26}$. The preheating result for $\sigma_{8}=0.94$ case again shows good agreement with $\mathrm{X}$-ray observations except for some excess luminosity at galaxy group scale with $T_{500}<1 \mathrm{keV}$. 

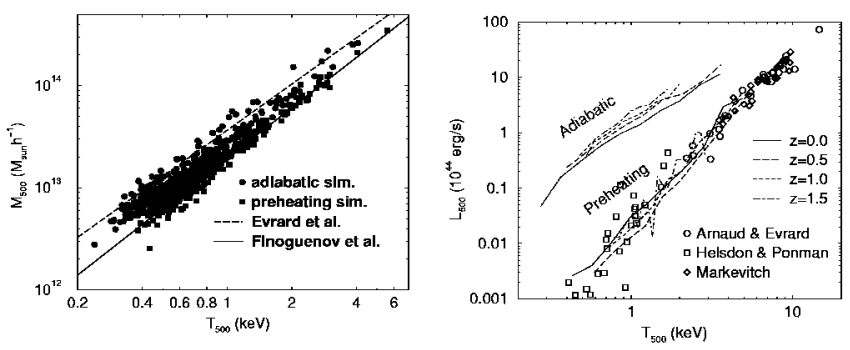

Fig. 1. (a) $M_{500}-T_{500}$ relation: Adiabatic (circles) and preheating (squares) simulation data for $\sigma_{8}=0.94$, to be compared with the another adiabatic simulation (long-dashed line), and the observational data fit (solid line). (b) $L_{500}-T_{500}$ relation: Adiabatic (shaded lines) and preheating (dark lines) simulation results at 4 different redshifts. Observation results are represented as different points.

\section{SZ and CMB Power Spectra}

The agreement of our $M-T$ and $L-T$ relations with observations assures the proposed heating scheme to be well-constrained by observations. We thus proceed to construct one- $\mathrm{deg}^{2} \mathrm{SZ}$ maps up to $z=2.5$, following the method described in da Silva et al. $(2000)^{27}$.

We employ the scaling formula in Komatsu \& Seljak (2002) $)^{28}$ and rescale the SZ power spectra of both $\sigma_{8}=1$ and 0.94 cases in Fig.2 from our original $\Omega_{b} h^{2}=0.018$ and 0.0218, respectively, to $\Omega_{b} h^{2}=0.022$ used in Bond et al. $(2002)^{15}$ for detailed comparison. The CBI deep-field observations scanned a $9 \mathrm{deg}^{2}$ sky ${ }^{13}$. We hence compute the preheated $\mathrm{SZ}$ power spectrum averaged over 9 one- $\mathrm{deg}^{2} \mathrm{SZ}$ maps and add it to the (noise-free) primary CMB spectrum of $\Omega_{b}=0.04, \Omega_{m}=0.3, \Omega_{\Lambda}=0.7$ used in Bond et al. $(2002)^{15}$, to obtain one realization. A total of 40 realizations are constructed for checking the SZ cosmic variance. Both $\sigma_{8}=0.94$ and 1 runs show that preheating reduces the averaged SZ power spectra by a factor $2-3$ over the angular scales $l=2000-4000$. Clear in Fig.2 is that the preheated SZ + CMB power spectrum of $\sigma_{8}=1$ lies well below the $2-\sigma$ error box of CBI data, but it is consistent with ACBAR data ${ }^{14}$.

Using the $\sigma_{8}{ }^{7}$ scaling law of Komatsu \& Seljak $(2002)^{28}$ to estimate the preheated SZ flux, we find that $\sigma_{8}$ must be raised to 1.2 for SZ effects to explain the CBI high- $l$ data. But $\Omega_{m}$ is then pushed down to $0.13 \pm 0.02$, constrained by $\sigma_{8} \Omega_{m}^{0.6}=0.35 \pm 0.03^{17}$. Such an $\Omega_{m}$ is too low for the Big-Bang nuclear synthesis model to be consistent with the observed X-ray baryon fraction $(\leq 1 / 4)$ in massive clusters ${ }^{29} 30$. Various explanations, other than SZ effects, for the CBI high- $l$ excess have also been proposed and they were discussed in Pearson et al.(2003) ${ }^{13}$. 


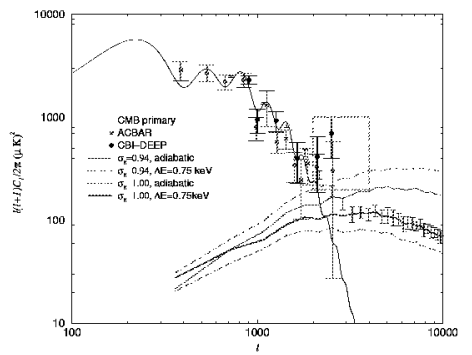

Fig. 2. Comparison of CBI and ACBAR data with the averaged preheating and adiabatic SZ power spectra of $\sigma_{8}=0.94 \& 1$, and with the CMB + preheated SZ spectrum of $\sigma_{8}=1$ where its 1- $\sigma$ error bars arise from the cosmic variance over 40 fields of $9 \mathrm{deg}^{2}$. While the $\sigma_{8}=1$ adiabatic SZ spectrum comfortably intersects the CBI $2-\sigma$ error box over the band $\mathrm{l}=2000-4000$, the preheating spectrum lies well below the CBI data.

\section{References}

1. Navarro, J., Frenk, C.S. \& White, S.D.M., ApJ, 462, 563, (1996)

2. Chiueh, T. \& He, X.G., Phys. Rev., D65, 123518 (2002)

3. Ponman, T.J., Bourner, P.D., Ebeling, H. and Böhringer, H., MNRAS,283, 690 (1996)

4. Allen, S.W. \& Fabian, A.C., MNRAS, 297, L57 (1998)

5. Xue, Y.J. \& Wu, X.P., ApJ, 538, 65 (2000)

6. Finoguenov, A., Reiprich, T. H., \& Böhringer, H., A\&A, 368, 749 (2001)

7. Tabor, G. \& Binney, J., MNRAS,263, 123 (1993)

8. David, L.P., et al., ApJ, 557, 546 (2001)

9. da Silva, A.C., et al., ApJ, 561, L15 (2001)

10. Bialek, J.J., Evrard, A.E. \& Mohr, J.J., ApJ, 555, 597 (2001)

11. Borgani, S., et al., MNRAS, 336, 409 (2002)

12. Muanwong, O., Thomas P.A., Kay, S.T, \& Pearce, F.R., MNRAS, 336, 527 (2002)

13. Pearson, T.J., et al., ApJ, 519, 556 (2003)

14. Kuo, C.L., et al., (2002) astro-ph/2012289

15. Bond, J.R. et al., (2002) astro-ph/0205386

16. Goldstein, J.H., et al., (2002) astro-ph/0212517

17. Bahcall, N.A. et al., ApJ, 585, 182 (2003)

18. Rosati, P., Borgani, S. \& Norman, C., ARAA, 40, 539 (2002)

19. White, M., Hernquist, L. \& Springel, V., ApJ, 579, 16 (2002)

20. Finoguenov, A., Jones, C., Böhringer, H. \& Ponman, T.J., ApJ, 578, 74 (2002)

21. Yamada, M. \& Fujita, Y., ApJ, 533, L145, (2001)

22. Springel, V., Yoshida, N. \& White, S.D.M., New Astronomy, 6, 79 (2001)

23. Evrard, A.E., Metzler, C.A. \& Navarro, J.F., ApJ, 469, 494 (1996)

24. Arnaud, M. \& Evrard, A. E., MNRAS, 305, 631 (1999)

25. Helsdon, S. F. \& Ponman, T. J., MNRAS, 319, 933 (2000)

26. Markevitch, M., ApJ, 504, 27 (1998)

27. da Silva, A.C., Barbosa, D., Liddle, A.R. \& Thomas, P.A., MNRAS, 317, 37 (2000)

28. Komatsu, E., \& Seljak, U., MNRAS, 336, 1256 (2002)

29. Ettori, S \& Fabian, A.C., MNRAS, 305, 843 (1999)

30. Mohr, J.J., Mathiesen, B.F. \& Evrard, A.E., ApJ, 517, 627 (1999) 